\title{
Miniature Microwave Bandpass Filter Based on EBG Structures
}

\author{
Zhurbenko, Vitaliy; Krozer, Viktor; Meincke, Peter
}

Published in:

Proceedings of the 36th European Microwave Conference

Link to article, DOI:

10.1109/EUMC.2006.281038

Publication date:

2006

Document Version

Publisher's PDF, also known as Version of record

Link back to DTU Orbit

Citation (APA):

Zhurbenko, V., Krozer, V., \& Meincke, P. (2006). Miniature Microwave Bandpass Filter Based on EBG Structures. In Proceedings of the 36th European Microwave Conference (pp. 792-794). IEEE.

https://doi.org/10.1109/EUMC.2006.281038

\section{General rights}

Copyright and moral rights for the publications made accessible in the public portal are retained by the authors and/or other copyright owners and it is a condition of accessing publications that users recognise and abide by the legal requirements associated with these rights.

- Users may download and print one copy of any publication from the public portal for the purpose of private study or research.

- You may not further distribute the material or use it for any profit-making activity or commercial gain

- You may freely distribute the URL identifying the publication in the public portal

If you believe that this document breaches copyright please contact us providing details, and we will remove access to the work immediately and investigate your claim. 


\title{
Miniature Microwave Bandpass Filter Based on EBG Structures
}

\author{
Vitaliy Zhurbenko, Viktor Krozer and Peter Meincke \\ Technical University of Denmark, Ørsted $\bullet$ DTU, Electromagnetic Systems, \\ Ørsteds Plads Building 348, 2800 Kgs. Lyngby, Denmark, Phone:+45-45253820, Fax: +45-45931634, \\ E-mail:vz@oersted.dtu.dk
}

\begin{abstract}
A new design of a planar microwave filter, based on rejection band properties of an electrically small electromagnetic bandgap (EBG) structure, is proposed. The proposed EBG structure demonstrates effective impedance manipulation, exhibits a simple analysis, and is about three times smaller as compared to stepped-impedance hairpin (SIH) resonators with similar response. The new bandpass filter has a reduced footprint and can be fabricated in standard thick-film manufacturing technology. Measured and simulated results exhibit good agreement. The measured results show improvement in the filter characteristics in comparison to existing SIH filter design.

Index Terms-Electromagnetic bandgap, microstrip,
\end{abstract} microwave filter, slow-wave effect.

\section{INTRODUCTION}

Com ompact, high-performance microwave filters are essential for high-efficiency miniaturized microwave systems. The filter circuit size is large in traditionally designed planar bandpass filters due to a high number of large area resonators. The rejection level in the upper stopband of the filters is usually degraded by the spurious response at twice the passband frequency. Several types of resonators have been designed to overcome these problems, such as miniaturized hairpin resonators, stepped-impedance hairpin resonators, and slow-wave open-loop resonators. Coupled transmission line sections are frequently used today for the realization of compact matching circuits to various devices $[1,2]$.

Miniaturized resonators lead to a reduced filter size, but not always improve the spurious response. Another method relies on various resonator realisations within one filter structure to reduce the circuit size, such as the coupled line filter with $\mathrm{SIH}$ resonator proposed by Wang et al. [3]. Two coupled line resonators loaded by the stepped-impedance hairpin resonator are employed in this filter. The performance of the filter can be significantly enhanced by carefully adjusting the load impedances of the coupled line sections.

The load impedance adjustment can also be achieved by implementing slow-wave EBG structures, which reduces the length of the microwave resonator and contributes to the filter miniaturization $[4,5]$.

This paper proposes an EBG structure exhibiting a lowpass, slow-wave characteristic for impedance manipulation of reduced size bandpass filters. A combination of slow-wave EBG structures and coupled line resonators leads to compact bandpass filters, exhibiting improved stopband characteristics. As an example this paper presents measured and simulated results for a high performance reduced dimension bandpass filter in comparison to existing filter types.

\section{Microstrip EBG Structure}

It is generally assumed that EBG structures give rise to large area filters, as has been shown by various groups $[6,7]$. However, compact slow-wave EBG structures can be realized in a form of a periodically loaded transmission line. The proposed pattern for a microstrip line is shown in Fig.1.

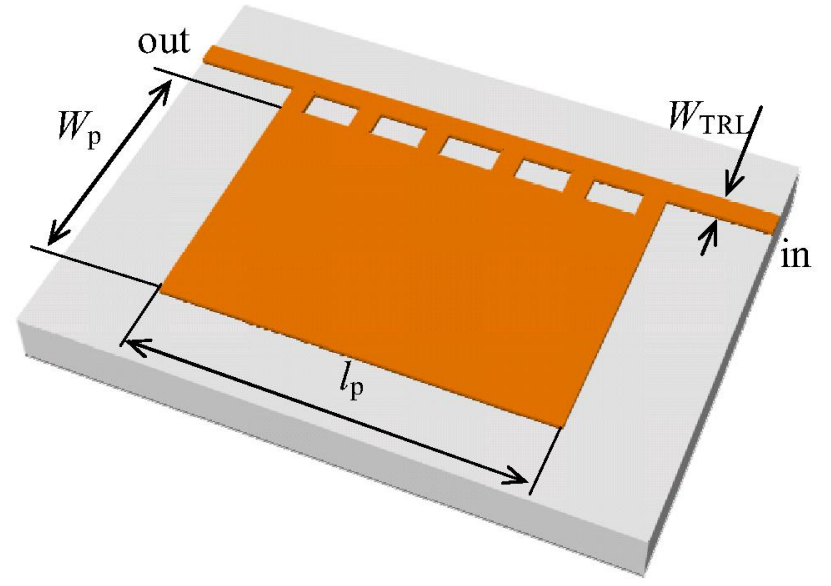

Fig. 1. Periodically loaded microstrip transmission line section used as a slow-wave EBG structure.

The concept of the structure behavior can be explained by considering relations for the transmission line. As can be seen in Fig. 1, the EBG structure consists on a high impedance microstrip line with characteristic impedance $Z_{\mathrm{TRL}}$ given by

$$
Z_{\mathrm{TRL}}=\sqrt{\frac{L_{\mathrm{TRL}}}{C_{\mathrm{TRL}}}},
$$

where $C_{\mathrm{TRL}}$ is the capacitance per unit length and $L_{\mathrm{TRL}}$ is the inductance per unit length of the line. The high impedance transmission line is loaded by a patch with width $W_{\mathrm{p}}$ and length $l_{\mathrm{p}}$ using short transversal stubs. In this configuration the most significant contribution to the equivalent capacitance of the EBG structure comes from the parallel plate capacitance of the patch, which can be obtained by

$$
C_{\mathrm{p}}=\frac{\varepsilon_{0} \varepsilon_{r} W_{\mathrm{p}}}{h},
$$

where $\varepsilon_{0}=8.85 \cdot 10^{-12} \mathrm{~F} / \mathrm{m}, \varepsilon_{r}$ is the relative permittivity, and $h$ is the thickness of the substrate in Fig. 1.

The characteristic impedance of the resulting structure can be estimated by: 


$$
Z_{\mathrm{EBG}}=\sqrt{\frac{L_{\mathrm{TRL}}}{C_{\mathrm{TRL}}+C_{\mathrm{p}}}}=Z_{\mathrm{TRL}} \sqrt{\frac{C_{\mathrm{TRL}}}{C_{\mathrm{TRL}}+C_{\mathrm{p}}}} .
$$

The quantities $C_{\mathrm{TRL}}$ and $Z_{\mathrm{TRL}}$ are calculated using the wellknown relations for microstrip lines. Obviously, $C_{\mathrm{p}}$ and $L_{\mathrm{TRL}}$ have the most significant influence on the characteristics of the EBG section. The inductance $L_{\mathrm{TRL}}$ is adjusted by varying the transmission line width $W_{\text {TRL }}$, whereas the capacitance $C_{\mathrm{p}}$ can be adjusted by varying the parameter $W_{\mathrm{p}}$. By tuning these parameters and the microstrip section length $l_{\mathrm{p}}$, different slowwave effects can be attained at different frequencies. The operating frequency bandwidth depends on the number of transversal stubs. The feeding lines, which connect the structure to the $50 \mathrm{Ohm} \mathrm{load,} \mathrm{should} \mathrm{be} \mathrm{also} \mathrm{taken} \mathrm{into}$ account because they have en influence on parameters of the structure.

An advantage of this EBG structure is that no etching nor via holes are required in the ground metallization or in the substrate, respectively. Therefore, this technique is compatible with planar fabrication techniques and simplifies the filter design process.

Using such a structure the self-capacitance and the selfinductance per unit length of the transmission line are increased, giving rise to a slow-wave effect. Due to this effect the microstrip device dimension can be significantly reduced. At the same time, it is possible to manipulate the line impedance. The effectiveness regarding the occupied area for a given impedance transformation is illustrated in Table 1.

$$
\text { TABLE } 1
$$

IMPEDANCE TRANSFORMATION RATIO OF THE EBG STRUCTURE

\begin{tabular}{l|c|c|c|c|c}
\hline Transformation ratio & $1: 2$ & $1: 3$ & $1: 4$ & $1: 5$ & $1: 6$ \\
\hline Length $/ \lambda$ & 0.13 & 0.13 & 0.13 & 0.13 & 0.13 \\
\hline Width $\lambda$ & 0.101 & 0.110 & 0.118 & 0.120 & 0.150 \\
\hline
\end{tabular}

$\lambda$ is the wavelength in the substrate material; number of shunt stubs is 4

The impedance transformer employing the EBG section is less than quarter wavelength in length and width and can be used for a wide range of impedance ratios. Indeed, it can be seen from Table 1 that different impedance transformation ratios are achieved with different widths $W_{\mathrm{p}}$ in the range of $0.1 \leq \lambda \leq 0.25$, while keeping the length and the number of shunt stubs constant.

The analysis of the proposed structure has been successfully accomplished using well-known transmission line models. The microstrip transmission-line models provide sufficient accuracy for the construction of the EBG structure in the frequency range considered here.

\section{BANDPAss Filter BASEd On COUPLED Line SECTIONS AND EBG STRUCTURE}

The new EBG structure is employed in the design of the bandpass filter shown in Fig. 2. This filter has the same center frequency and bandwidth as that presented in [3]. The filter of [3], however, based on SIH resonator instead of an EBG structure. The SIH resonator implemented in [3] improves the selectivity and eliminates the $2^{\text {nd }}$-harmonic response of coupled-line sections simultaneously. Filter circuits were implemented on a FR-4 substrate with $\varepsilon_{\mathrm{r}}=4.4$ and thickness $\mathrm{h}=0.745 \mathrm{~mm}$. Fig. 3(a) illustrates measured results for the transmission and return loss characteristics of this filter.
The return loss characteristics can be significantly improved by reducing the impedance of the coupled lines and increasing the coupling level. This means that the coupled lines width $\left(\mathrm{W}_{\mathrm{c}}=0.2 \mathrm{~mm}\right)$ or the separation between the lines $(\mathrm{S}=0.2 \mathrm{~mm})$ have to be reduced even further, which makes the fabrication process more difficult decreases reproducibility.

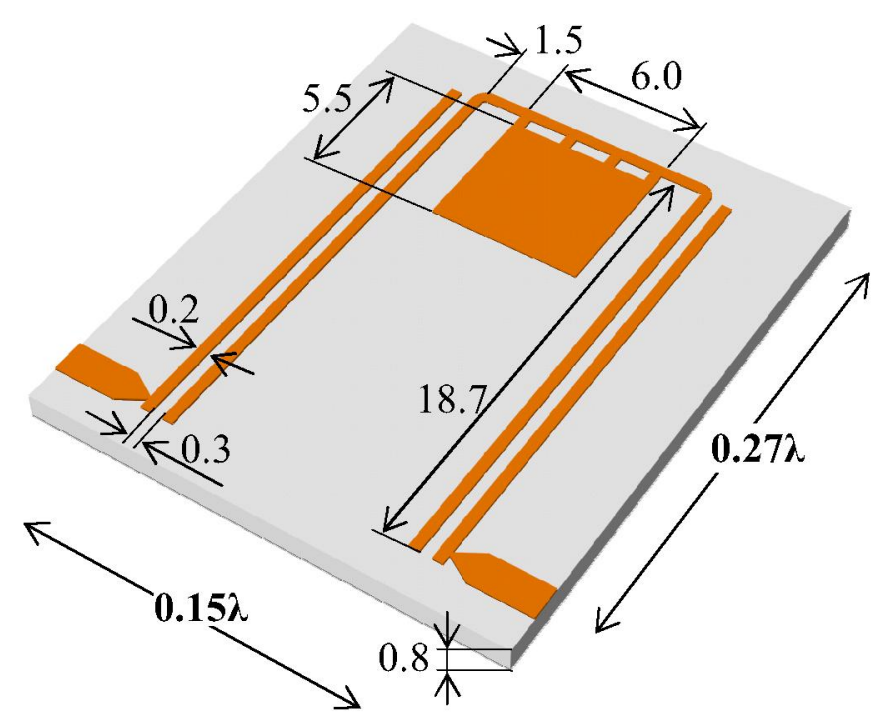

Fig. 2. Schematic of the coupled line bandpass filter with EBG structure (dimensions in $\mathrm{mm}$ )

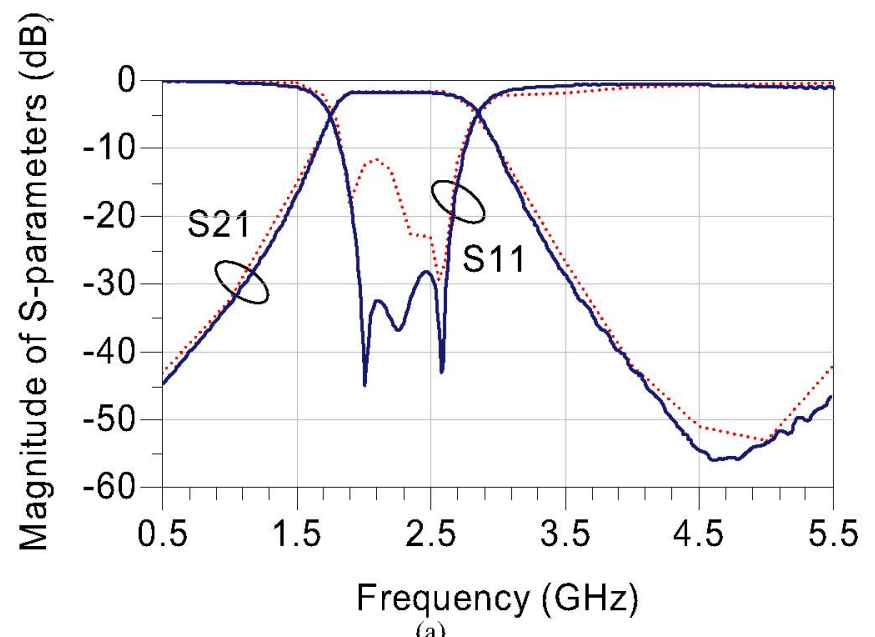

(a)

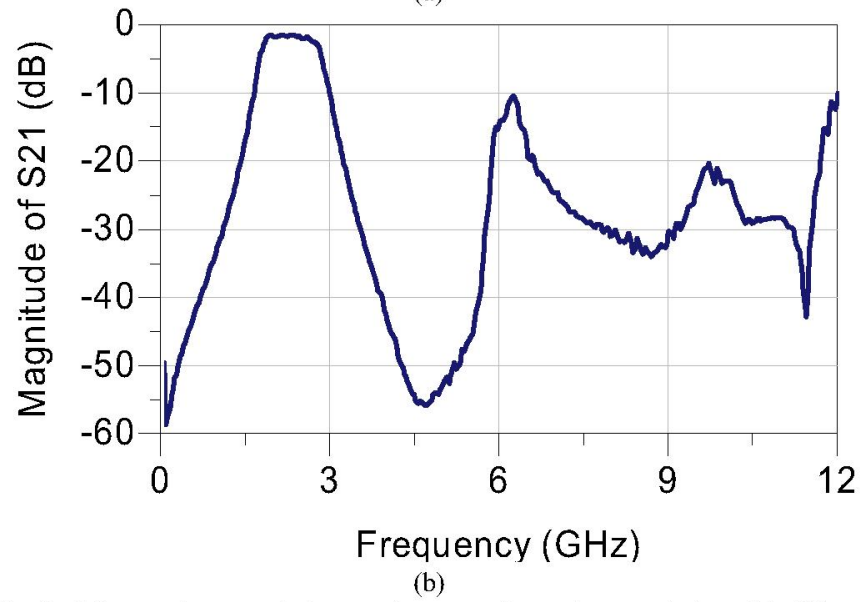

Fig. 3. Measured transmission and return loss characteristics. (a) Filters comparison. The dotted line: existing (SIR based filter) design; the solid line: proposed (EBG based filter) design. (b) Spurious response. 
An alternative way is to change the load of coupled lines by replacing the SIH resonator with the proposed EBG structure, as shown in Fig. 2. In this configuration the load impedance of the coupled lines can easily be adjusted for improved filter matching.

As mentioned above the EBG structure exhibits low-pass characteristics. Due to this fact, the $2^{\text {nd }}$-harmonic response is effectively suppressed. This is because the EBG as well as the SIH structure give rise to the same parasitic effect to increase the shunt capacitance. The selectivity of the filter at high frequencies is controlled by the EBG sections, whereas the coupled line sections control the low frequency response.

The proposed filter in Fig. 2 is designed using empirical models for single and coupled microstrip lines. Empirical models for coupled microstrip lines are not as accurate as those for single lines [8], since the mutual inductance and the mutual capacitance are not easily predictable.

An accurate prediction of the coupled lines response is carried out using method of moments simulations. The device was fabricated on the same substrate as the traditional filter mentioned above [3].

The EBG structure employed in the filter is three times smaller as compared to the SIH resonator. The new filter has therefore a footprint area reduced by about $30 \%$ as compared to the filter proposed in [3]. The overall dimensions of the filter are approximately $0.15 \lambda \times 0.27 \lambda$, where $\lambda$ is the guided wavelength in the substrate at center frequency.

Measurement results for the proposed filter and the one discussed in [3] are shown in Fig. 3 and Fig.4. It can be seen from Fig. 3a that the implementation of the EBG structure in Fig. 1 improves the matching of the device. Also, the $2^{\text {nd }}$-harmonic response is effectively suppressed. The passband insertion loss is about $1.5 \mathrm{~dB}$, including the connector and the tapped feed line loss. The selectivity of the filter can be defined as

$$
\xi=\left|\frac{\alpha_{\min }-\alpha_{\max }}{f_{s}-f_{p}}\right|\left(\frac{\mathrm{dB}}{\mathrm{GHz}}\right),
$$

where $f_{s}$ is the stopband frequency at $\alpha_{\text {min }}$ with a $20-\mathrm{dB}$ attenuation point, and $f_{p}$ is the $3-\mathrm{dB}$ cutoff frequency at $\alpha_{\max }$ with a 3-dB attenuation point. The lower- and upper-band selectivity of the new bandpass filter is improved to better than $43 \mathrm{~dB} / \mathrm{GHz}$ and $38 \mathrm{~dB} / \mathrm{GHz}$, respectively. The $2^{\text {nd }}$-harmonic response of the proposed structure is improved from $-53 \mathrm{~dB}$ to $-55.6 \mathrm{~dB}$. The filter has center frequency $f_{0}=2.3 \mathrm{GHz}$ and a first spurious response at about $2.7 f_{0}=6.2$ $\mathrm{GHz}$. At this spurious frequency, the minimum insertion loss is found to be $10.5 \mathrm{~dB}$, as shown in Fig. $3 b$.

The group delay is an important performance aspect for a wide-band filter, especially for filters used for processing of signals with complex frequency content. Fig. 4 shows a plot of the group delay within the filter bandpass region.

The designed structure achieves a smooth delay pattern in the operating frequency band with a variation of the order of $600 \mathrm{ps}$. Taking advantage of this property, the device can be effectively used as a component in a variety of systems requiring linear phase characteristics.

The developed filter is intended to be used in a microwave

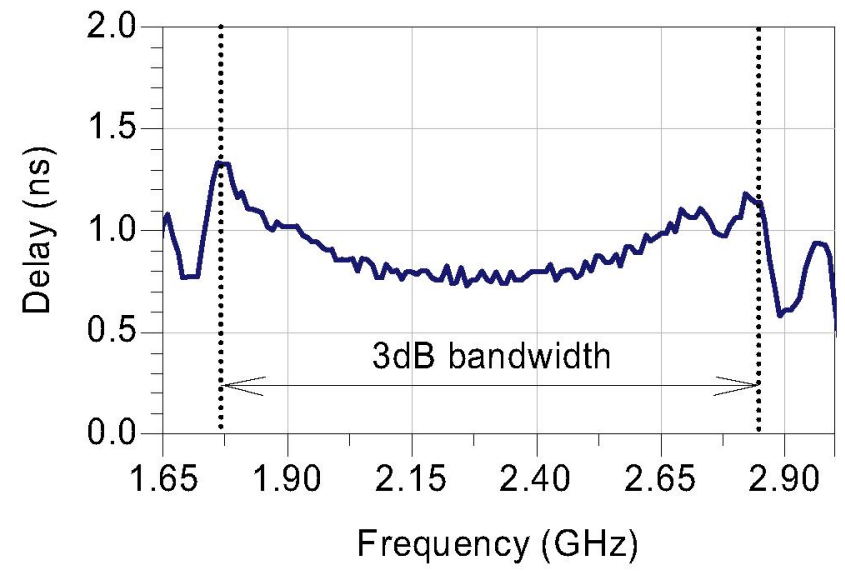

Fig. 4. Measured group delay.

camera for medical applications.

\section{CONClusion}

The development of microstrip circuits using EBG structures and coupled lines is promising for the development of compact high performance microwave components. A filter has been designed and fabricated for the demonstration of the compactness and improved performance of this method technique. The new filter exhibits improved matching characteristics and suppression of the harmonic response in the stopband. It also reduces the overall geometrical dimensions of the circuit. The fabrication technology is simple, inexpensive, and does not require via-holes or etching in a ground plane. An improvement in the $2^{\text {nd }}$ harmonic suppression has been achieved reaching a measured value of $-55.6 \mathrm{~dB}$. The filter exhibits a $3 \mathrm{~dB}$ bandwidth of $45 \%$ and an insertion loss of $1.5 \mathrm{~dB}$, a return loss in the passband of better than $25 \mathrm{~dB}$, with good phase linearity, leading to a group delay of \pm 300 ps.

\section{REFERENCES}

[1] K. S. Ang, C. H. Lee, and Y. C. Leong, "Analysis and design of coupled line impedance transformers," IEEE MTT-S Digest, pp. 1951-1954, 2004.

[2] G. Jaworski, V. Krozer, “A design of feeding network for a dual-linear polarization, stacked, probe-fed microstrip patch antenna array," Microwaves, Radar and Wireless Communications, vol. 2, pp. 473-476, May 2004.

[3] Yu-Zhen Wang, Man-Long Her, Yi-Chyun Chiou, and Ying-De Wu, "New coupled-line bandpass filter with stepped impedance hairpin (SIH) resonator," Microwave And Optical Technology Letters, vol.44, no.1, pp. 80-83, January 52005.

[4] H. Lin, J. Mao, and J. Liu, "A novel compact K band PBG microstrip structure band-pass filter," International Journal Of Electronics, vol. 92, no. 9 , pp. $467-472,2005$

[5] D. Nesic, "Compact one-cell EBG (or PBG) microstrip matching line," Microwave And Optical Technology Letters, vol. 44, no. 4, pp. 363-365, February 2005

[6] I. Rumsey, M. Piket-May, P. K. Kelly, "Photonic Bandgap Structures Used as Filters in Microstrip Circuits," IEEE Microwave and Guided Wave Letters, vol. 8, no. 10, pp. 336-338, Oct 1998.

[7] M. Tong, Y. Lu, Y. Chen, M. Yang at al.," Design and analysis of planar printed microwave and PBG filters using an FDTD method," Microelectronics Journal, no. 35, pp. 777-781, 2004.

[8] S. A. Maas, The RF and microwave circuit design cookbook. Norwood: Artech House microwave library, 1998 